\title{
Foreword
}

\section{Not Such a Trivial Pursuit}

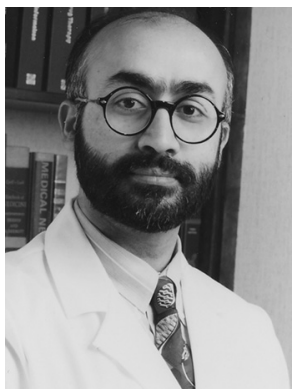

Ranjan K. Thakur, MD, MPH, MBA, FHRS

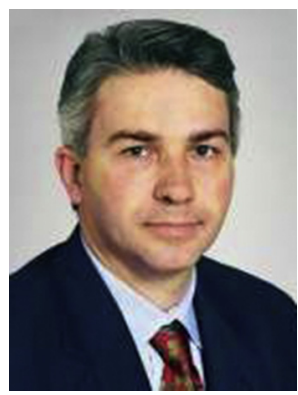

Andrea Natale, MD, FACC, FHRS

Editors

In cardiac electrophysiology we often face challenging cases that require deliberate application of electrophysiological principles to ascertain arrhythmia mechanisms and determine where ablation should be targeted. We initially acquire these skills from our teachers in the electrophysiology laboratory during fellowship training or going over complex tracings one-on-one.

A successful electrophysiology career requires that we continue practicing these skills as consultants, but we still need to keep learning and relearning by discussing difficult cases with our colleagues and reading monographs like the one Drs Shenasa and Gerstenfeld have put together to illustrate the established core principles for electrocardiographic and electrophysiologic diagnosis of complex arrhythmias.

This issue of Cardiac Electrophysiology Clinics deals with important complex cases that electrophysiologists don't see every day; therefore, they could easily forget some important principles that may be critical. The reader will find articles written by thought leaders in specific arrhythmias, which summarize the known important principles in dealing with these arrhythmias. Some examples include articles on fascicular tachycardias, epicardial arrhythmias, arrhythmias in congenital heart disease, electrocardiographic analysis of paced rhythms and their correlation with intracardiac electrograms, electrocardiographic characteristics of ventricular tachycardias in right ventricular dysplasia, and so on.

These arrhythmias are not encountered routinely in clinical practice. For this reason, it is important to review and refresh our understanding of important principles in diagnosis and management of these arrhythmias periodically. We congratulate Drs Shenasa and Gerstenfeld for assembling a thoughtful table of contents that will be helpful for fellows in training as well as clinical electrophysiologists in practice. In addition, we congratulate them for assembling a formidable list of contributors who draw upon decades of study and research to illuminate these issues authoritatively.

Ranjan K. Thakur, MD, MPH, MBA, FHRS Sparrow Thoracic and Cardiovascular Institute Michigan State University 1200 East Michigan Avenue, Suite 580 Lansing, MI 48912, USA

Andrea Natale, MD, FACC, FHRS Texas Cardiac Arrhythmia Institute

Center for Atrial Fibrillation at

St. David's Medical Center 1015 East 32nd Street, Suite 516

Austin, TX 78705, USA

E-mail addresses:

thakur@msu.edu (R.K. Thakur) andrea.natale@stdavids.com (A. Natale) 